\title{
Análisis mecánico del brazo de biela mediante norma ASTM e8
}

\section{Mechanical analysis of the connecting rod arm by ASTM e8 standard}

Guillermo Gorky Reyes Campaña

Fausto Cañizares

Ana Villacres

Marco Vinicio Noroña

Universidad Internacional del Ecuador, Ecuador

Autor por Correspondencia: manoroname@internacional.edu.ec, gureyesca@internacional.edu.ec, mdarioh@ hotmail.com, abelen@hotmail.com

Fecha de recepción: 3 de Febrero de 2017 - Fecha de aceptación: 28 de Abril de 2017

Resumen: Dos brazos de biela fueron analizados, uno nuevo y uno usado. Ambos pertenecientes al vehículo de marca Isuzu modelo Trooper año 1984. El objetivo fue determinar las propiedades mecánicas y las cargas máximas que puede soportar el elemento. Para esto, se realizaron ensayos de tracción bajo la norma ASTM E8, ensayos de compresión y un ensayo de carga a la rotura en la Máquina universal de ensayos que tiene una capacidad de 120.000 lbf/60 TON. Los resultados mostraron que el material de los brazos de biela es un acero AISI 4340 OQT 1300. Las propiedades mecánicas del elemento mejoraron con el tiempo y la temperatura de funcionamiento del motor debido al tratamiento térmico de normalización. En caso de que los esfuerzos aplicados al brazo de biela superen las cargas máximas registradas, el elemento puede sufrir una falla o deformación. A partir de los análisis realizados se determinó que el brazo de biela usado se encuentra en condiciones óptimas para ser utilizado en una reparación de motor. Asegurando así el adecuado funcionamiento y evitando el reemplazo del elemento, lo que da como resultado un ahorro económico.

Palabras clave: brazo de biela; ASTM E8; ensayo; acero AISI 4340; esfuerzo

\begin{abstract}
Two connecting rods were analyzed, one new and one second-hand. Both belong to the 1984 Trooper model from Isuzu. The research goal was to determine the mechanic properties as well as the maximum loads that this element can handle. Following the aim, tensile tests were conducted following the guidelines ASTM E8, also compression essays and a loading test for rupture in the universal machine with a capacity of $120.000 \mathrm{lbf} / 60 \mathrm{TON}$. According with the results, the material from the connecting rod is steel AISI 4340 OQT 1300. The mechanic properties of the connecting rod showed improvement due to thermic normalization when the time and temperature of the engine increased. In case that the stress applied to the connecting rod is above the maximum load, the item could have a failure or be deformed. According to the essays conducted in this research project, the connecting rod is in optimum conditions to be used for an engine repairment. This, ensuring a good performance of the element and avoiding the item replacement which results also in economical savings.
\end{abstract}

Key words: connecting rod; ASTM E8; essays; steel AISI 4340; stress 


\section{Introducción}

En los talleres mecánicos generalmente la reparación de los motores conlleva a la verificación del estado de todas las piezas móviles y fijas del motor antes de la realización de un trabajo de rectificación, pero en la mayoría de ocasiones los brazos de biela son reutilizados sin una previa revisión de su estado o de un análisis de sus propiedades mecánicas. Un estudio realizado en una empresa dedicada a la importación y comercialización de maquinarias de rectificación de motores automotrices indica que el kilometraje aproximado para que un motor necesite reparación es a los $300.000 \mathrm{~km}$ y un vehículo liviano alcanzaría este recorrido alrededor de los 7 años de uso [1], estos valores también van a depender del cuidado y buen mantenimiento que se dé al vehículo; caso contrario esto va influir en el desgaste anticipado de los elementos fijos y móviles de un motor, ya que sobre cada elemento del motor actúan fuerzas o momentos derivados de las condiciones físicas al momento de la combustión como la presión de los gases y a las masas inerciales que están inmersas en un motor de combustión interna [2]. Según una investigación sobre los desechos producidos en la rectificación de un motor de combustión interna se rectifican 21 brazos de biela en un mes [3], lo que significa que estos elementos no son cambiados, ya que los brazos de biela están diseñados para tener una vida infinita a la fatiga debido a que este elemento está sometido a millones de repeticiones de ciclos de carga, esta información fue obtenida de un análisis de la fractura de una biela de un compresor de refrigeración [4].

La biela es un elemento fundamental en el funcionamiento de un motor ya que trasforma la energía química generada por la combustión en una energía cinética, siendo el enlace entre la absorción de la energía mecánica, por lo que la biela es el elemento más tensionado de un motor [4]. Este elemento genera esfuerzos de tracción, compresión y flexión al momento de realizar su ciclo termodinámico de trabajo, debido a estos esfuerzos la biela es robusta en su diseño pero a la vez lo más ligera posible para reducir la inercia y soportar los esfuerzos mecánicos a los que se encuentra sometida [5], se fabrican por fundición de hierro o forjado por estampación de hierro al carbono aleado con otros materiales, posterior a estos procesos se mecanizan los taladros de engrase y los orificios del bulón y el cigüeñal [5].

No es muy común que una biela falle pero a través de diferentes trabajos de investigación se determina diferentes causas o motivos del porque bielas han sufrido algún tipo de falla, esto sucede cuando supera la tensión de fluencia del material y sufre un pandeo plástico que ocasiona la ruptura del elemento [6]; también puede fallar por fatiga que es ocasionada por defectos en la microestructura del material o por el excesivo desgaste del elemento, otra falla se genera por problemas tribológicos entre la biela y el cigüeñal generando un desgaste severo por el enorme aumento del coeficiente de fricción [4]. Una biela arqueada o retorcida sobre su eje provoca un ruido desmesurado en el motor y este arqueamiento es ocasionado por un diámetro excesivo en el pie o cabeza de la biela, por un ancho de la cabeza de biela deficiente o por un peso inadecuado del elemento [5].

El objetivo de este estudio es el de analizar un brazo de biela de un motor que ha alcanzado el kilometraje necesario para una reparación y no presenta ninguna falla en su estructura, y comparar esto con el análisis de un brazo de biela nuevo; como se observa en la figura 1 la biela que se analizó pertenece a un vehículo Trooper de motor Isuzu G200 y el 
material empleado en su fabricación es acero al carbono aleado con níquel y cromo, este elemento es fabricado por estampación al caliente y tiene mecanizadas las zonas de unión con el embolo y el cigüeñal [7]. Para desarrollar este análisis se realizó un ensayo de tracción bajo la norma ASTM E8 a probetas normadas de las dos muestras a comparar, con el fin de obtener los valores del límite de fluencia y la resistencia a la tracción del material del que está hecho el elemento. Finalmente se realizó ensayos de compresión a muestras obtenidas de la biela nueva y usada; y un ensayo de carga a una biela usada para determinar en ambos casos la carga máxima registrada.

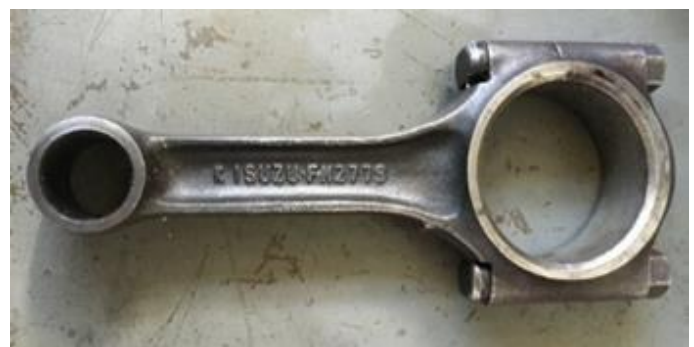

Figura 1. Brazo de biela motor Isuzu G200.

Fuente: Autores

\section{Fundamento teórico}

\section{Material}

El brazo de biela es uno de los primeros elementos que absorbe la energía mecánica, por lo que necesita una estabilidad mecánica para obtener dicha resistencia; son fabricados generalmente de aceros templados, éstos son, aceros semiduros y aceros aleados [8], que en este caso de estudio están constituidos de acero al carbono aleado con níquel y cromo, ya que este tipo de biela es la más común en el ámbito automotriz [9]. El brazo de biela soporta altas cargas compresivas por ello el elemento que ayuda a cumplir con las exigencias de trabajo es el cromo cuya propiedad mecánica es aumentar la dureza y dar un alto grado de resistencia al calor en el proceso cíclico térmico del motor [10], dando así una mejora en la templabilidad y alta resistencia al desgaste [9]; otro de los componentes constitutivos en el brazo de biela es el níquel que aleado con el acero permite obtener alta tenacidad en la biela, pero cuya desventaja sería la fragilidad de Krupp que a temperaturas entre 450 y $550^{\circ} \mathrm{C}$ tiene como consecuencia la disminución de la tenacidad provocando fragilidad en el brazo de biela [11], por tal motivo la aleación está conformada de otro componente como el molibdeno, cuya propiedad mecánica ayuda a disminuir dicha fragilidad y a soportar las temperaturas generadas en el proceso del motor.

El material del cual está fabricada la biela objeto de este estudio es de acero al carbono aleado con níquel y cromo [7]; según el sistema de designación AISI se usa cuatro dígitos para aceros al carbón y aceros aleados, los dos primeros dígitos señalan el grupo específico de aleaciones que identifica a los principales elementos aleantes aparte del carbono en el acero y los últimos dos dígitos indican la cantidad de carbono en el acero [12]. Para aceros aleados con níquel, cromo y molibdeno se usa la designación 43xx que tiene como composición química: $1.8 \%$ de níquel, $0.5-0.8 \%$ de cromo, $0.25 \%$ de molibdeno y $0.40 \%$ de carbón [12]; los aceros al medio carbón contienen de 30 a 50 puntos de carbono y presentan una resistencia de moderada a 
alta, una ductibilidad buena y una dureza moderada, por esta razón el acero AISI 4340 corresponde al material de la biela en estudio ya que este tipo de acero se aplica en engranajes, ejes y piezas que requieren buen endurecimiento interior como la biela [12].

\section{Esfuerzos}

Las fuerzas que actúan en el motor de combustión interna, son las llamadas fuerzas alternativas de inercia, se dividen en las de primer orden mediante el cambio de velocidad del pistón cuando pasa del PMS al PMI, y las de segundo orden que existen debido a la aceleración que posee el pistón [13], estas fuerzas se dan por medio del proceso cíclico no constante de la fuerza que ejerce el gas y de las piezas que poseen movimientos alternativos [14], en éste caso de estudio la longitud de la biela desempeña un papel importante ya que por su diseño alargado provoca el fenómeno de pandeo [15], por ésta razón se busca reducir dicha longitud para disminuir los efectos inerciales [16]. Otra de las fuerzas que están inmersas en el motor, son las fuerzas centrífugas que se generan debido al movimiento de las masas rotarias y alternativas [13]; en las masas alternativas se tiene el pistón, segmentos, pie de biela y cuerpo de biela cuyo movimiento se da de una forma alternativa en los 2/3 del cuerpo de biela [13] según lo indicado en la figura 2; y las masas rotatorias como la cabeza de biela con tapa de cojinete, tornillos, tuercas, muñequilla y cuerpo de biela que posee un movimiento rotativo en el 1/3 del cuerpo de biela [13].

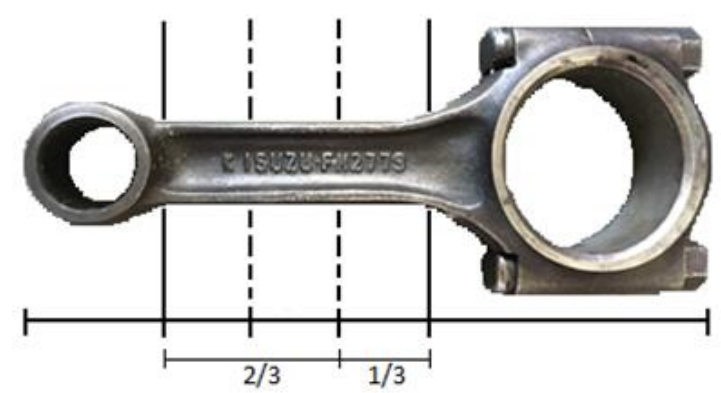

Figura 2. Masas alternativas y rotatorias en el brazo de biela. Fuente: Autores

Los esfuerzos son aplicados de forma irregular en la biela, por ejemplo el de tracción y compresión actúan en el pie de biela; para disminuir la fuerza de compresión se toma en cuenta las dimensiones de sección en el brazo de biela [15]. Cuando se transmite las fuerzas del émbolo al cigüeñal, se generan por la inclinación los esfuerzos de tracción, compresión y flexión [15] que actúan en la cabeza de biela; las cargas de flexión, compresión y flexión lateral se aplican en el cuerpo de biela, que a su vez soporta el pandeo [16]. Para contrarrestar la flexión y tenga una adecuada resistencia se basa en la forma [15] y esta dependerá de la inercia que tenga el elemento, ya que en el movimiento del pistón se ejerce una fuerza de inercia que se transfiere desde la mitad superior del cojinete del bulón hacia la biela, provocando una fuerza de tracción en los pernos del elemento a estudiar y un esfuerzo de compresión en la parte inferior de los cojinetes de muñequilla del cigüeñal [17]; por esta razón la biela tiene forma en I, para que obtenga igual resistencia a la flexión que una sección rectangular con idéntica longitud, la desventaja es que en la sección en I se genera fenómenos de pandeo y para remediar esta desventaja se procede a colocar nervios $\mathrm{N}$ [13]. 


\section{Dinámica del mecanismo de biela y manivela}

Durante el funcionamiento del motor, en el mecanismo biela-manivela, mostrado en la figura 3, actúan fuerzas derivadas de la presión de los gases y de la inercia de las masas en movimiento [18]; el análisis de estas fuerzas es indispensable para realizar los cálculos de la resistencia mecánica de las piezas del motor [18], en concordancia con el método cinetostatico las fuerzas de fricción se desprecian [18]. Las fuerzas de inercia que influyen en las masas del mecanismo poseen velocidades variables en magnitud y dirección [19], estas fuerzas están inmersas en todo el ciclo de trabajo del motor y se dividen en fuerzas con movimiento alternativo y giratorio [18]. Las fuerzas de inercia superan considerablemente a las de los gases ya que los motores desarrollan un gran número de revoluciones, y por esta razón son las más importantes al momento de realizar los cálculos de resistencia a la fatiga de las piezas [19].

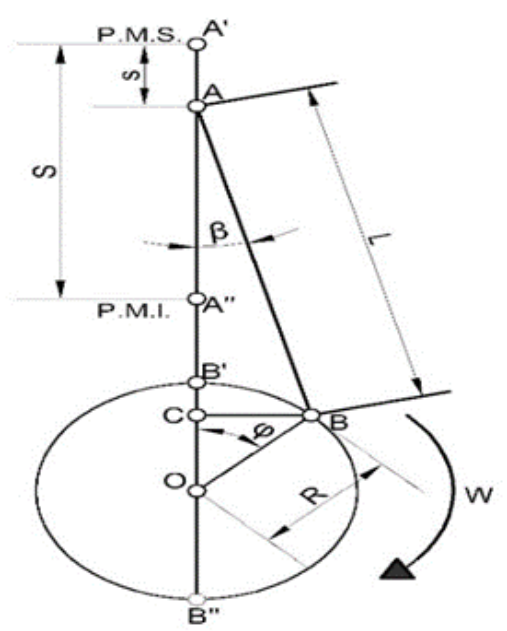

Figura 3. Esquema del mecanismo biela-manivela. Fuente: [18]

Para calcular la fuerza de biela es importante conocer la fuerza que ejerce el gas sobre el émbolo, cuyo valor es inversamente proporcional al ángulo de inclinación [14].

F_S $=$ F_G $/ \cos \beta$

F_G: Fuerza que ejerce el gas sobre el émbolo

$\beta$ : Angulo de inclinación de la biela

Las masas del mecanismo de biela-manivela poseen fuerzas de inercia [19] que se dividen:

$\checkmark$ Fuerzas de inercia de las masas que se mueven alternativamente.

$\checkmark$ Fuerzas de inercia de las masas que giran.

$\checkmark$ Fuerzas de inercia de las masas que efectúan un movimiento complejo. 


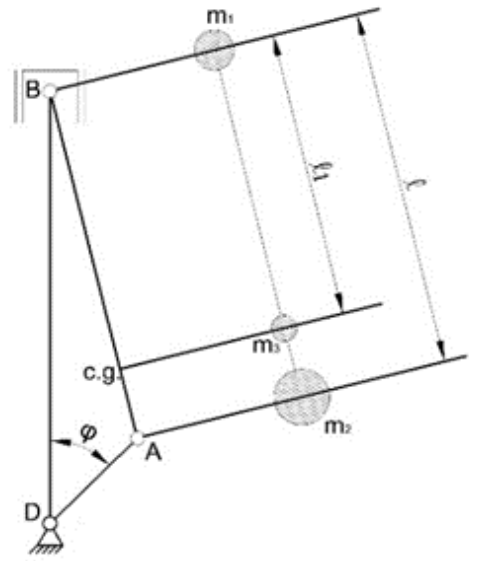

Figura 4. Reducción de las masas. Fuente: [19]

La biela posee un movimiento de avance y vaivén en el plano perpendicular al eje del cigüeñal [19] y para determinar los valores de las fuerzas de inercia y los cálculos dinámicos del mecanismo de la biela de una manera más simple, se utiliza el método de la reducción de la masa [19] descrito en la figura 4, en la cual se divide la biela en tres masas; la masa m1 se concentra en el eje del pie de biela, la $\mathrm{m} 2$ en el eje de la cabeza y la $\mathrm{m} 3$ en su centro de gravedad para guardar la dinámica del mecanismo [19]. Para que este método mantenga la semejanza con la dinámica de la biela en el sistema de tres masas, la suma de las tres masas será igual a la masa de la biela $\mathrm{mb}$, el centro de gravedad común de las tres masas coincidirá con el centro de gravedad de la biela y las masas se encontrarán en una recta que pase por el centro de gravedad de la biela [19].

$$
\mathrm{mb}=\mathrm{m} 1+\mathrm{m} 2+\mathrm{m} 3[19]
$$

\section{Metodología}

El brazo de biela que se utilizó para realizar los respectivos ensayos es de un vehículo de marca Isuzu, Modelo Trooper año 1984 el cual posee un kilometraje de 1’197.599 y su cilindrada es de $2.000 \mathrm{cc}$. Debido a que estos vehículos fueron vendidos a partir del año 1980, se afirma que en la actualidad han recorrido más de $300.000 \mathrm{Km}$, lo que asegura que estos vehículos poseen un brazo de biela apto para este estudio y así generar la comparativa con el elemento nuevo. Visualmente se observó que los brazos de biela nuevos y usados no presentaban ningún desperfecto en su estructura externa como se aprecia en la figura 5, además se verificó que las dimensiones de las bielas usadas y nuevas son idénticas; y el peso era muy similar tanto en la biela nueva como en la biela usada que presentaban valores de $680,39 \mathrm{~g}$ y de $657,70 \mathrm{~g}$ respectivamente. 


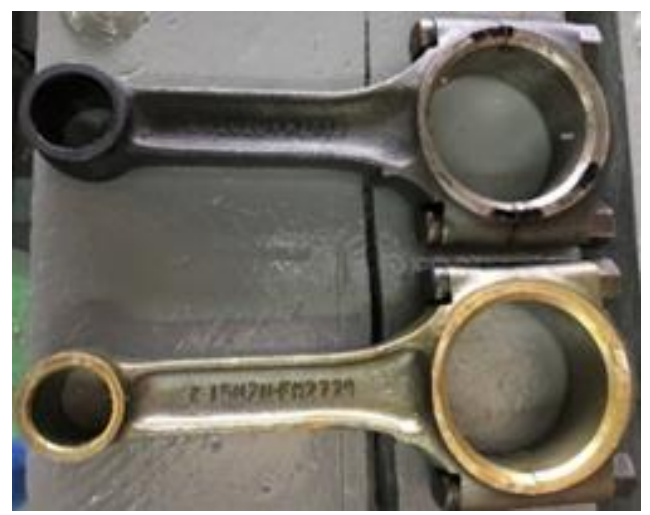

Figura 5. Biela usada y nueva del vehiculó Fuente: Autores

Los ensayos se realizaron en una Máquina universal de ensayos, marca Tinius Olsen modelo Super L120, serie 224875 y con una capacidad de 120.000 lbf/60 TON.

Se realizó el ensayo de tracción con el fin de determinar las características mecánicas del material, a probetas obtenidas de las bielas nueva y usada de acuerdo a la Norma ASTM E8 [20] como se observa en la figura 6.

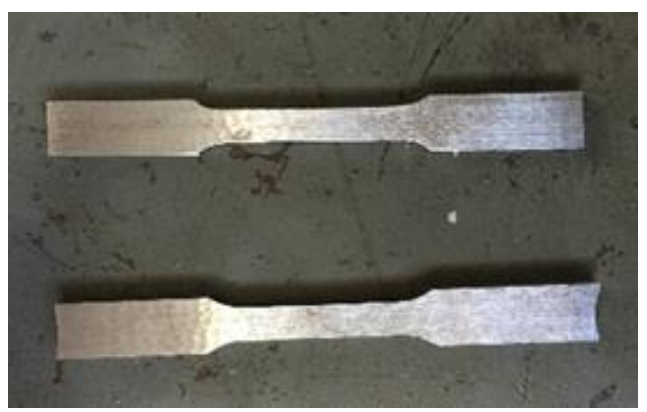

Figura 6. Probetas para ensayo de tracción bajo la Norma ASTM E8. Fuente: Autores

Para el ensayo de compresión se utilizó muestras de las bielas nueva y usada, las cuales se obtuvieron mediante el corte transversal de la cabeza y el pie de biela, consiguiendo como muestra final netamente el cuerpo de biela como se muestra en la figura 7 , debido a que esta parte del elemento está sometido a una compresión por la fuerza que se produce en el instante de la combustión [18]. Este estudio fue con la finalidad de obtener la carga máxima a la cual se someterá el brazo de biela y ver la deformación que sufrió el elemento cuando está sometido a un esfuerzo de compresión. 


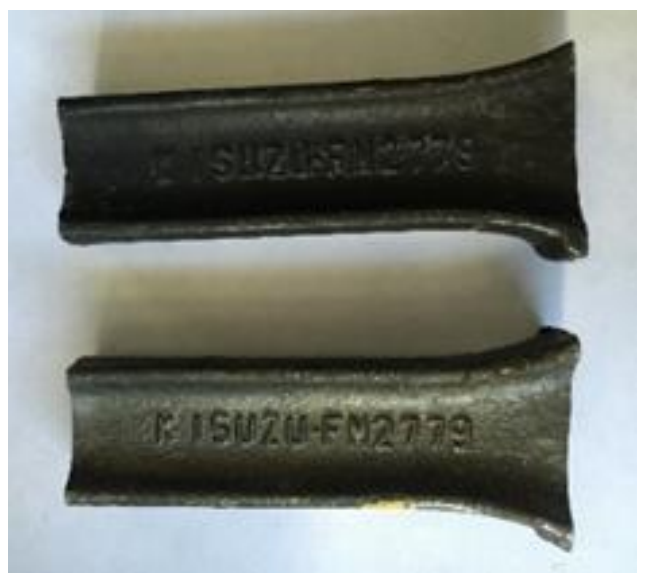

Figura 7. Muestras para ensayo de compresión.

Fuente: Autores

En el procedimiento del ensayo de carga se utilizó una biela usada, en este caso el elemento completo se sujetó a la maquina universal de ensayos mediante cables en sus extremos como se aprecia en la figura 8 , y se aplicó fuerza de tracción para determinar la carga máxima que soportó el brazo de biela; se ocupó el elemento completo debido a que en el funcionamiento del motor todo el brazo de biela se encuentra sometido a esfuerzos.

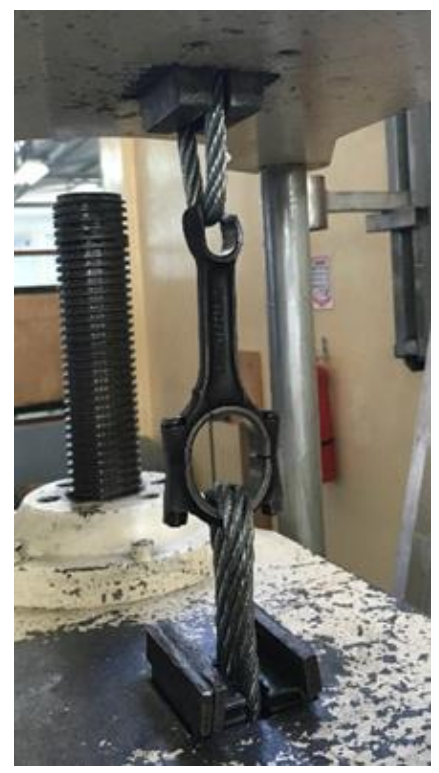

Figura 8. Ensayo de carga a brazo de biela Fuente: Autores

Para la obtención de los datos de los diferentes tipos de ensayos se obtuvo como variable independiente el material ya que este no cambio luego de la aplicación de cargas a las diferentes probetas y muestras utilizadas, otra variable independiente son las probetas que se realizaron bajo la norma ASTM E8, ya que la normativa indica los valores establecidos del tamaño de la muestra. Obteniendo como variables dependientes los esfuerzos o cargas aplicados a las muestras que tuvieron como resultado valores distintos, y las bielas ya que en la realización de los ensayos cambiaron su estructura molecular y mecánica. 


\section{Análisis y discusión}

\section{Ensayo de tracción}

Finalizado el ensayo se obtuvieron los valores cuantitativos mostrados en la tabla 1 de las propiedades mecánicas del elemento.

Tabla 1. Resultados del ensayo de tracción.

\begin{tabular}{ll|l}
\hline Propiedades & Biela nueva & Biela usada \\
\hline $\begin{array}{l}\text { Carga máxima } \\
\text { registrada (N) }\end{array}$ & 16.331 & 17.963 \\
$\begin{array}{l}\text { Límite de fluencia } \\
\text { (MPa) }\end{array}$ & 849,54 & 927,58 \\
$\begin{array}{l}\text { Resistencia a la } \\
\text { tracción (MPa) }\end{array}$ & 971,02 & 1079,50 \\
$\begin{array}{l}\% \text { Elongación en } \\
\text { 25mm }\end{array}$ & 17,00 & 20,88 \\
\hline
\end{tabular}

Fuente: Laboratorio EPN

El material que corresponde a los brazos de biela es un acero AISI 4340 y tienen valores de resistencia a la tracción entre (745 - $1950 \mathrm{MPa})$ y un límite de fluencia entre (469 - 1570 $\mathrm{MPa}$ ) [12]; las bielas en su fabricación están sometidas a tratamiento térmicos de normalización, temple y revenido [18], estos tratamientos ocasionan que los valores de las propiedades mecánicas varíen. Con los resultados obtenidos del ensayo del límite de fluencia y de resistencia a la tracción, se determinó que estos valores son similares a los valores teóricos del acero AISI 4340 OQT 1300 [12] que se observan en la tabla 2; este tipo de acero tiene un tratamiento inmerso en aceite y templado a $1300^{\circ} \mathrm{F}$ [12], lo que significa que este material corresponde al utilizado en la fabricación de los brazos de biela estudiados.

Tabla 2. Propiedades mecánicas del acero AISI 4340 OQT 1300.

\begin{tabular}{l|l}
\hline Propiedades Mecanicas & Valor \\
\hline Resistencia A La Tracción: & $965 \mathrm{Mpa}$ \\
Resistencia Al Punto Cedente: & $827 \mathrm{Mpa}$ \\
Ductibilidad (Elongación & 23 \\
Porcentual En 2 Pulg): & $280 \mathrm{Hb}$ \\
Dureza Brinell: & $7680 \mathrm{Kg} / \mathrm{M}^{3}$ \\
Densidad: & $207 \mathrm{Gpa}$ \\
Módulo De Elasticidad: & 0.27 \\
Razón De Poisson: & $80 \mathrm{Gpa}$ \\
Módulo De Corte: & $6.5 \times 10^{-6}{ }^{\circ} \mathrm{F}^{-1}$ \\
Coeficiente De Expansión & \\
Térmica: &
\end{tabular}

Fuente [12]

Los resultados detallados en la tabla1 de los ensayos que se realizaron a las probetas, indican que los valores del límite de fluencia y de resistencia a la tracción de la probeta de la biela usada están por encima de los valores de la probeta de la biela nueva como se explica en la figura 9. 


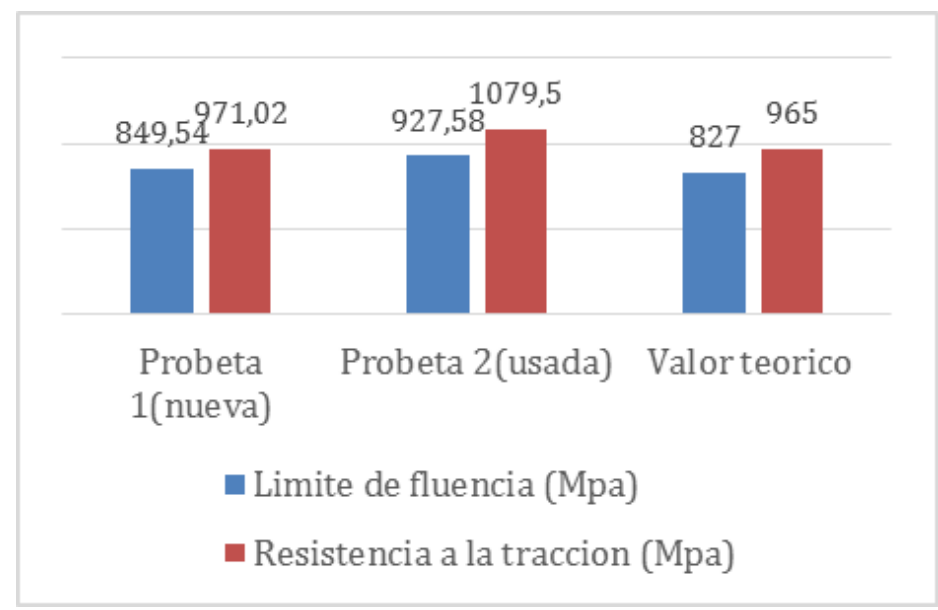

Figura 9. Resultados del ensayo de tracción. Fuente: Autores

Analizando los resultados y conociendo que la biela usada durante su vida útil estuvo sometida a temperaturas de funcionamiento entre $\left(800-950^{\circ} \mathrm{C}\right)$ [7], se determinó que la estructura del material durante el tiempo de funcionamiento del motor, sufrió un tratamiento térmico de normalización, lo que ocasionó que el elemento usado tenga una mejor estructura interna y una mayor resistencia [12], ya que en el normalizado el material se calienta a una temperatura aproximada de $870^{\circ} \mathrm{C}$ [12], se mantiene en esta temperatura y posterior se enfría a temperatura ambiente [21], este tratamiento refina los granos de perlita, deshace la red de cementita, y eleva las propiedades mecánicas del acero [21].

\section{Ensayo de compresión}

En el ensayo se aplicó un esfuerzo en el eje longitudinal del cuerpo de biela, para simular las condiciones de trabajo del componente en el motor [6] obteniendo los resultados mostrados en la tabla 3.

Tabla 3. Resultados del ensayo de compresión.

\begin{tabular}{lllll}
\hline & & $\begin{array}{l}\text { Biela } \\
\text { nueva }\end{array}$ & $\begin{array}{l}\text { Biela } \\
\text { usada }\end{array}$ \\
\hline $\begin{array}{l}\text { Carga máxima } \\
\text { registrada }\end{array}$ & $\mathrm{lbf}$ & 42.321 & 35.989 \\
& $\mathrm{~N}$ & 188.253 & 160.087 \\
$\begin{array}{l}\text { Longitud } \\
(\mathrm{mm})\end{array}$ & $\begin{array}{l}\text { Antes del } \\
\text { ensayo }\end{array}$ & 75 & 75 \\
& $\begin{array}{l}\text { Después } \\
\text { del ensayo }\end{array}$ & 72,95 & 73,85 \\
\hline
\end{tabular}

Fuente: Laboratorio EPN

La prueba permitió verificar la carga de compresión aplicada de una manera progresiva [22] en cada una de las muestras del brazo de biela nueva y usada, puesto que se visualizó el ensayo de una manera directa, el modo de deformación de las muestras es de forma curveada [23] como se observa en la figura 10. La combinación de los ciclos de carga a los que se 
encuentra sometido el brazo de biela ocasiona que un modo de falla de este elemento sea el pandeo, fenómeno que es tenido en cuenta en el diseño de una biela [6]. Para que el fenómeno de pandeo exista el material supera el límite elástico o límite de fluencia [6] y la carga critica de pandeo, esta carga es en función de la geometría de la pieza y el módulo de elasticidad del material [6].

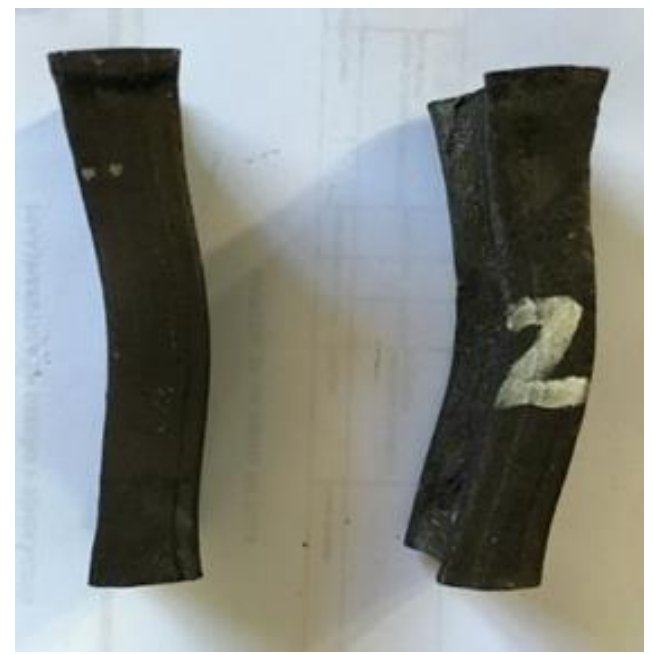

Figura 10. Muestras después del ensayo de compresión. Fuente: Autores

Con los resultados obtenidos del ensayo se estableció que la muestra de la biela nueva soporto más carga como se distingue en la figura 11 y sufrió un mayor deformación; debido al tratamiento térmico de templado que presenta el material de la biela nueva, soporta más carga de compresión ya que al transformar su estructura de austentita en martensita obtiene una dureza más alta y una mejor solidez en el elemento [21].

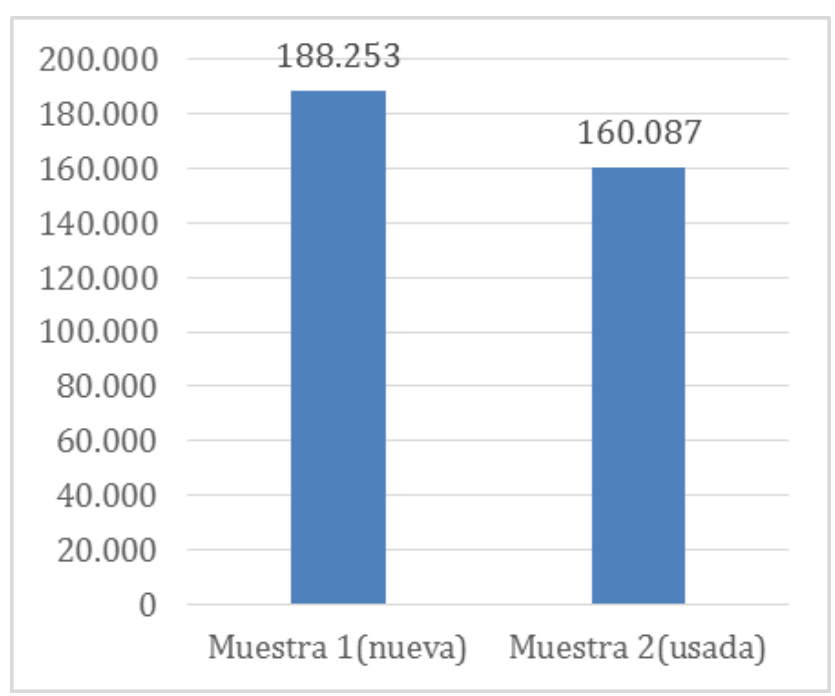

Figura 11. Resultados de la carga máxima registrada en el ensayo de compresión. Fuente: Autores

De acuerdo a los resultados del ensayo se comprobó que la temperatura afecto notablemente las propiedades mecánicas de la biela usada, ya que todos los materiales presentan 
una menor resistencia a la deformación a temperaturas elevadas [24]. Cuando se aumenta la temperatura de trabajo en el brazo de biela, se reduce la resistencia a la fluencia [24] y se incrementa la ductilidad, este cambio de propiedades es importante ya que cualquier operación de deformación se alcanza a temperaturas elevadas con menor fuerza y potencia [24].

\section{Ensayo de carga}

La fuerza aplicada en el ensayo de carga a la rotura es mayor a la del ensayo de tracción, por ende aumentan las velocidades de fluencia ya que en la prueba se conduce hasta la rotura del material [25].

Tabla 4. Resultados del ensayo de carga.

\begin{tabular}{|c|c|c|c|}
\hline & & & $\begin{array}{ll} & \text { Biela } \\
\text { usada } & \\
\end{array}$ \\
\hline \multirow{2}{*}{ máxima } & Carga & lbf & 21.765 \\
\hline & & $\mathrm{N}$ & 96.816 \\
\hline & Longitud & Antes & 189,5 \\
\hline$(\mathrm{mm})$ & & Después & 204,1 \\
\hline \multicolumn{2}{|r|}{ Observaciones } & & $\begin{array}{l}\text { Fallo } \\
\text { pie de biela }\end{array}$ \\
\hline
\end{tabular}

Fuente: Laboratorio EPN

Finalizado el ensayo la probeta cedió por fractura en el pie de biela y el valor de su longitud se incrementó como se indica en la tabla 4, por lo que se comprueba que la deformación total de la muestra en ciertos casos llega hasta el 50\% de su estructura [25].

Se verificó que la parte más frágil del elemento es el pie de biela porque es el segmento que presenta una menor área, la disminución de ésta área se conoce como estricción [26] y es donde se ocasiona la deformación, esto provoca que la capacidad de la biela en la cual se está aplicando la carga disminuya [26], y exista una menor resistencia a la carga aplicada. Consecuentemente la parte que no se fracturo resistió debido a que presenta un área mayor, y esto permitió que la fuerza aplicada se distribuya uniformemente, de igual manera la sujeción de los pernos ayudó a que la carga aplicada no ocasione la fractura en la cabeza de biela [26].

\section{Conclusiones}

A partir de los resultados obtenidos y el análisis de los ensayos realizados, los valores prácticos de las propiedades mecánicas de los brazos de biela como el límite de fluencia con valores de 849,54 y 927,58 MPa y resistencia a la tracción con valores de 971,02 y 1079,50 MPa, cumplen con los datos teóricos del tipo de material del cual están fabricadas. A su vez, se demostró que el brazo de biela de un vehículo con un recorrido de 1'197.599km, mejoró su estructura y sus propiedades mecánicas debido a que la temperatura de los ciclos de trabajo del motor que van entre los 800 y $950{ }^{\circ} \mathrm{C}$, ocasionaron en el elemento el tratamiento térmico de normalización dando al material una mayor resistencia a la tracción con un valor de 1079,50 $\mathrm{MPa}$. 
El fenómeno de pandeo en una biela es una falla poco común que se evita si el elemento no es sometido a cargas mayores a los $160.000 \mathrm{~N}$ y hagan que el material sobrepase su límite de fluencia de $827 \mathrm{MPa}$. La biela tendrá un adecuado funcionamiento y no sufrirá alguna falla o deformación, si las cargas inerciales y los esfuerzos a los que se encuentra sometida durante el funcionamiento del motor, no superan a los valores de las cargas máximas registradas en los ensayos de compresión (160.087 - $188.253 \mathrm{~N})$ y de carga a la rotura $(96.816 \mathrm{~N})$.

Los resultados de los ensayos determinan que las propiedades mecánicas de límite de fluencia y resistencia a la tracción están dentro del rango permitido de $827 \mathrm{MPa}$ y $965 \mathrm{MPa}$ respectivamente para un óptimo funcionamiento, y debido a que cumple con dichas propiedades tanto en su límite plástico y elástico, el brazo de biela usado resulta apto para cumplir su función en una futura reparación del motor.

Debido al cambio de la matriz productiva, los entes estatales responsables de identificar los sectores productivos, y en este caso buscando la implementación de industrias para la fabricación de brazos de biela, cuyo resultado daría como beneficio la fomentación de trabajo utilizando la mano de obra ecuatoriana calificada; se lograría la viabilidad de esta idea con la implementación de tecnología en las industrias de metalurgia y siderurgia en el país.

\section{Bibliografía}

A. V. Jácome Gallegos, «Proyecto de factibilidad para la creación de la empresa asociativa Irsamotor dedicada a la importación y comercialización de maquinarias de rectificación de motores automotrices en la ciudad de Quito - Ecuador,» Mayo 2015. [En línea]. Available: http://dspace.ups.edu.ec/handle/123456789/9481.

C. Romero, H. Quintero y G. Trujillo, «Modelados de las cargas dinámicas en los motores de combustión interna,» Diciembre 2003. [En línea]. Available: http://revistas.utp.edu.co/index.php/revistaciencia/article/view/7391/4391.

A. B. Anrango Catucuamba, «Estudio y clasificación de los desechos de la rectificación de un motor de combustión interna,» 2013. [En línea]. Available: http://repositorio.espe.edu.ec:8080/handle/21000/6413.

E. Porras, S. Rodriguez y J. Coronado, «Análisis de la fractura de una biela de compresor de refrigeración,» Abril $2010 . \quad$ [En línea]. Available: http://www.scielo.org.co/pdf/iei/v30n1/v30n1a22.pdf.

S. Escudero, J. Gonzalez, J. Rivas y A. Suarez, Motores, Madrid: MACMILLAN Profesional, 2009.

C. Cravino, G. Cardus, H. Svoboda y H. De Rosa, «Evaluación de la falla de una biela de automotor,» 2003. [En línea]. http://www.sm2000.coppe.ufrj.br/mirror/sarra/artigos/artigo10229/10229.pdf.

M. Quezada y S. Seade, «Mantenimiento y comprobación de un motor Isuzu G 200,» 2006. [En línea]. Available: http://dspace.uazuay.edu.ec/handle/datos/366. 
S. Jara y V. Ordoñez, «Guia para repotenciación de motores a carburador con aplicación práctica en un motor $\mathrm{G}$ 10,» 2013. [En línea]. Available: http://dspace.uazuay.edu.ec/handle/datos/2217.

S. Barroso Herrero y J. Ibáñez Ulargui, Introducción al conocimiento de materiales, Madrid: UNED cuadernos, 2014.

H. Appold, K. Feiler, A. Reinhard y P. Schmidt, Tecnología de los metales para profesionales técnico - mecánicas, Barcelona: Editorial Reverté, 1984.

P. Molera Solá, Tratamiento térmico de los metales, Barcelona: BOIXAREU Editores, 1991.

R. Mott, Diseño de elementos de máquinas - Segunda edición, USA: Macmillan Inc., 1992.

D. González Calleja, Motores, Madrid: Ediciones Paraninfo, 2011.

Bosch, Manual de la técnica del automóvil - Tercera Edición, Barcelona: Editorial Reverté S.A., 1996.

E. CEAC, Manual CEAC del automóvil, Barcelona: Grupo editorial CEAC, 2004.

J. Álvarez, I. Callejón y S. Forns, Motores alternativos de combustión interna, Barcelona: Ediciones UPC, 2005.

E. Kates y W. Luck, Motores Diésel y de gas de alta compresión - Segunda Edición, Barcelona: Editorial Reverté S.A., 1981.

M. Jóvaj, Motores de automóvil, Moscú: Editorial MIR, 1982.

M. Jóvaj, Motores de automóvil, Moscú: Editorial MIR, 1973.

A. International, «E8/E8M - Standard test methods for tension testing of metallic materials, » 2009. [En línea]. Available: https://www.researchgate.net/file.PostFileLoader.html?id=56eb722993553bbbe15bbe8f\& assetKey=AS\%3A340826430427147\%401458270761896.

A. Malishev, G. Nikolaiev y Y. Shuvalov, Tecnología de losmetales - Sexta edición, Moscú: Editorial MIR.

A. Leyensetter, Tecnología de los oficios metalúrgicos, Barcelona: Editorial Reverté, 2006.

J. Newell, Ciencia de materiales Aplicaciones en ingeniería, México D.F.: Alfa omega, 2011.

M. Groover, Fundamentos de manufactura moderna - Materiales, procesos y sistemas, Estado de México: PEARSON Prentice Hall, 1997. 
F. Morral, E. Jimeno y P. Molera, Metalurgia general Tomo II, Barcelona: Editorial Reverté, S.A., 2004.

W. J. Callister, Introducción a la Ciencia e Ingeniería de los Materiales 1, Barcelona: Editorial Reverte, 2007. 